\title{
Duplicidad no Ectópica del Uréter: Implicancias Clínicas
}

\author{
Non-ectopic Duplicity of the Ureter: Clinical Implications.
}

Garbelotti Junior, S. A. ${ }^{1}$; Rodrigues Pereira, . $^{2}$; Marques, S. R. ${ }^{1}$; Moraes de Carvalho, L. O. ${ }^{1}$ \& Olave, E. ${ }^{3}$

GARBELOTTI JUNIOR, S.A.; RODRIGUES PEREIRA, V.; MARQUES, S. R.; MORAES DE CARVALHO, L. O. \& OLAVE, E. Duplicidad no ectópica del uréter: implicancias clínicas. Int. J. Morphol., 35(2):611-614, 2017.

RESUMEN: La duplicación del sistema colector renal es la variación más frecuente del sistema urinário y se puede presentar como duplicación completa o incompleta, así como la inserción ectópica en todas las partes del sistema urinario a partir de la parte distal hacia la vejiga. Este artículo presenta uma duplicación completa no ectópica del ureter en el riñón izquierdo de un cadáver de un individuo brasileño, de sexo masculino. Ambos uréteres tenían origen en el hilio renal y continuaban hasta la vejiga urinaria separadamente, desembocando en ostios diferentes, en el área del trígono vesical, donde el ostio perteneciente al uréter que drenaba el polo superior presentó posición distal y lateral en relación al ostio del uréter que drenaba el polo inferior. Esta disposición es uma excepción a la regla de Weigert-Meyer, que indica que el uréter del polo superior, por el hecho de permanecer fijo por más tiempo al conducto mesonéfrico, presenta mayor migración, terminando medial e inferiormente al uréter que drena el polo inferior en $97 \%$ de los casos.

PALABRAS CLAVE: Anatomía; Sistema urinario; Duplicación Uréter.

\section{INTRODUCCIÓN}

Las vías urinarias se inician en los cálices renales menores que se unen para formar dos o tres cálices renales mayores, los que se reunen para formar la pelvis renal, una dilatación infundibuliforme que sobrepasa el seno renal y se continúa com la parte abdominal del uréter que alcanza a la pelvis (parte pélvica del uréter) y pasa a través de la pared de la vejiga urinaria (parte intramural del uréter) y termina en los ostios ureterales (Goss, 1988; Moore et al., 2014; Standring, 2015).

Los uréteres son tubos que transportan la orina desde la pelvis renal hasta la vejiga urinaria. Sus paredes son espesas, donde su diámetro varía entre 1,0 hasta $10 \mathrm{~mm}$; su longitud va desde 28 hasta $34 \mathrm{~cm}$, siendo el uréter derecho cerca de 1,0 cm más corto que el izquierdo (Goss; Standring; Moore et al.). Los uréteres provienen del mesonefros (cuerpo de Wolff) encontrado en los segmentos torácico y lumbar superior, está constituído por um canal retorcido en $\mathrm{S}$ que se abre para el ducto mesonéfrico. Gradualmente las extremidades craneales de los túbulos desaparecen y nuevos túbulos se originan en la extremidad caudal. Una sección del ducto del riñón, incluyendo los túbulos colectores, cálices, pelvis renal y uréter, se desarrolla a partir del boton ureteral (Dorko et al., 2016).
La duplicación parcial o completa del uréter, triplicación o muy raramente cuadruplicación, se han descrito en la literatura, pero pocos trabajos muestran registros de mediciones o biometría (Urdangarain et al., 2006).

Entre las variaciones numéricas del tracto urinario superior, se encuentra la duplicación ureteral, la que se presenta en aproximadamente $0,8 \%$ de la población y ocurre dos veces más en la mujer que en el hombre, siendo bilateral en 15 $\%$ de los casos (Chacko et al., 2007; Alberts et al., 2013; Chao, 2013; Ellerkamp et al., 2013), además de eso, $10 \%$ son diagnosticados tangencialmente en niños investigados por afecciones del tracto urinario o infección presente en las mujeres con incontinencia urinaria (Faion et al., 2003; Chacko et al.; Senel et al., 2015). Son pocos los pacientes que necesitan de intervención quirúrgica debido a la duplicación ureteral, ya que en estos pacientes, los hallazgos típicos son ureterocele obstructivo, reflujo vesicoureteral grave y/o uréter ectópico, muchas veces en combinación con una porción de tejido renal no funcional (Ellerkamp et al.; Senel et al.).

La duplicación del uréter que ocurre con mayor frecuencia es la incompleta, donde los dos uréteres se unen próximo al tercio distal, resultando en un único uréter que alcanza

${ }^{1}$ Departamento de Morfologia e Genetica da Universidade Federal de São Paulo UNIFESP, São Paulo, Brasil.

${ }^{2}$ Disciplina de Anatomia Humana do Centro Universitário São Camilo - CUSC, São Paulo, Brasil.

${ }^{3}$ Facultad de Medicina, Universidad de La Frontera, Temuco, Chile. 
la vejiga, recibiendo la denominación de uréter bífido en "Y". En muchos casos, el uréter que drena el polo superior es ectópico y se encuentra obstruído aumentando el riesgo de reflujo y pielonefritis (Senel et al.; Urdangarain et al.).

El presente artículo presenta un caso de duplicación del uréter izquierdo en un cadáver de sexo masculino con registros biométricos y topográficos de esas estructuras, tratando de contribuir con clínicos y cirujanos, con el análisis de otros casos presentados por diversos investigadores y entregar datos comparativos para la imagenología.

\section{DESCRIPCIÓN}

En disección de rutina, se encontró en un riñón izquierdo de un cadáver de un individuo Brasileño, adulto, de sexo masculino, una duplicación completa no ectópica del uréter (Figs. 1 y 2). Los registros biométricos del uréter y riñón fueron los siguientes: la longitud del rinón desde el polo superior al inferior fue de $110,69 \mathrm{~mm}$, su espesor fue de $44,42 \mathrm{~mm}$ y la abertura del hilio renal fue de $24,9 \mathrm{~mm}$. Los uréteres fueron descritos como: superior (proveniente del polo superior, Fig. 1), el cual tuvo una longitud de $287 \mathrm{~mm}$ y un diámetro de 2,52 $\mathrm{mm}$ e inferior (proveniente del polo inferior, Fig.1), el que midió $273 \mathrm{~mm}$ de longitud y 3,9 mm de diámetro.

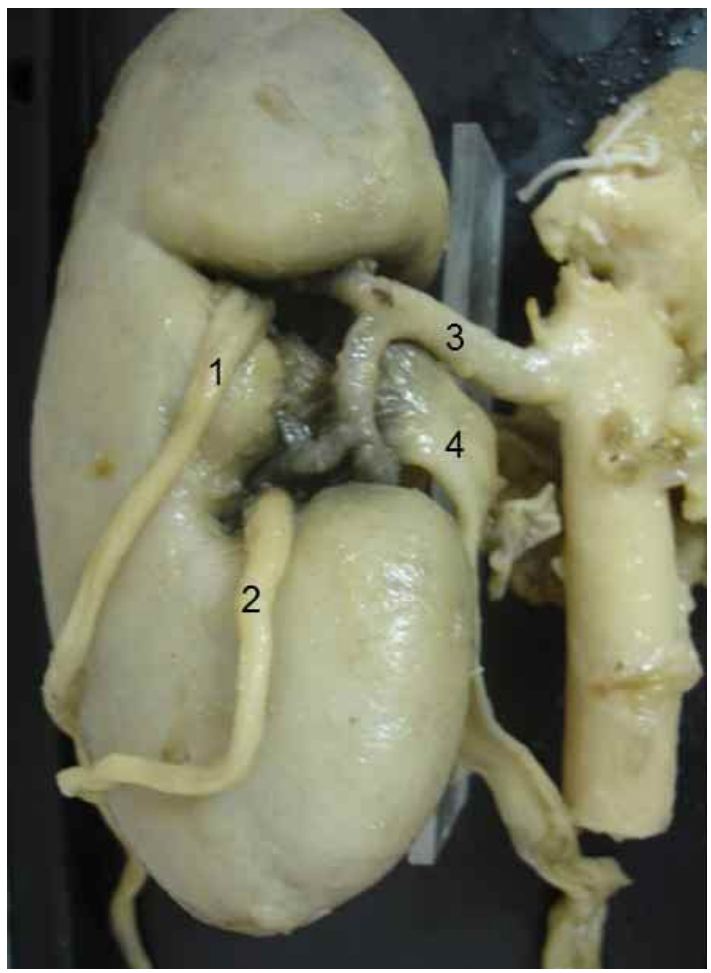

Fig. 1. Vista posterior del riñón izquierdo. 1. Uréter superior, 2. Uréter inferior, 3. arteria renal, 4. Vena renal.
Ambos uréteres tenían origen en el hilio renal y continuaron separados hasta la vejiga urinaria (Fig. 2), desembocando en ostios diferentes, en el área del trígono de la vejiga con una distancia entre ambos de $3,16 \mathrm{~mm}$, lo que caracterizó la variación de no ectópica, donde el ostio perteneciente al uréter superior presentó una posición distal y lateral respecto al ostio del uréter inferior (Fig. 3).

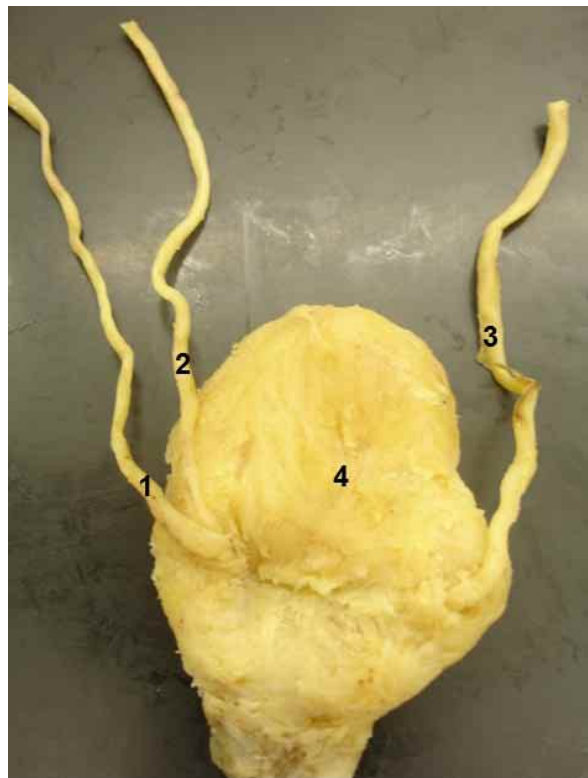

Fig. 2. Vista posterior de la vejiga urinaria. 1. Uréter superior, 2. Uréter inferior, 3. Uréter derecho, 4. Vejiga urinaria.

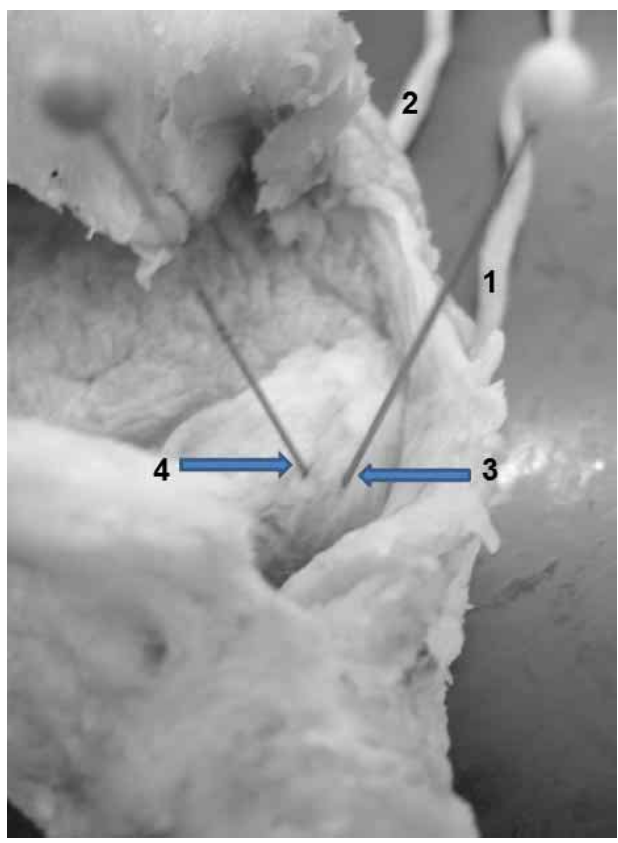

Fig. 3. Vista interna de la vejiga urinaria con ostios ureterales del lado izquierdo. 1. Uréter superior, 2. Uréter inferior, 3. Ostio del uréter superior, 4. Ostio del uréter inferior. 


\section{DISCUSIÓN}

Desde el punto de vista embriológico, estas anomalías urológicas resultan de la división precoz del brote ureteral embrionario y de los tejidos metanéfricos que son una estructura primordial del uréter y de la pelvis renal, resultando que la división incompleta del botón uretérico resulta en un uréter bífido. Para completar un sistema duplicado surgen dos brotes ureterales (Dorko et al.; Chao).

Según la regla de Weigert-Meyer, citada por Dorko et al. y Faion et al., el uréter del polo superior, por permanecer fijo al ducto mesonéfrico por más tiempo, presenta mayor migración, terminando medial e inferiormente al uréter que drena el polo inferior en el $97 \%$ de los casos, de tal modo que este caso se torna una excepción a la regla, ya que el uréter proveniente del polo superior se abre inferiormente pero lateral al del polo inferior. Además, el hecho del uréter superior migrar excesivamente puede tornarlo ectópico y eventualmente obstruído, mientras que el uréter que drena el polo inferior puede presentar un corto túnel intravesical, lo que puede resultar en reflujo vesicoureteral, siendo frecuentemente indicado el tratamiento quirúrgico precoz (Faion et al.; Lam, 1991; Choi \& Oh, 2000; Chao).

Además de eso, la duplicidad presentada fue hallada en un cadáver masculino, lo que según Dorko et al. es menos frecuente, ya que esta variación se presenta más en el sexo femenino en proporción de 2:1.

Esta disposición en general no causa problemas, pero en algunos casos uno de los orificios ureterales es ectópico, localizándose fuera del trígono de la vejiga. Este tipo de uréter ocurre más frecuentemente en mujeres (Senel et al.). En contraste, un uréter simple ectópico es más común en hombres y en esos casos, es alto el porcentaje (80 \%) de duplicación ureteral contralateral (Dorko et al.).

El uréter doble ocurre en $0,8 \%$ de la población (Ellerkamp et al.; Chacko et al.; Alberts et al; Chao) y raramente, en cerca del $1 \%$ de los casos diagnosticados, un ramo del uréter supernumerario puede terminar a ciegas causando obstrucción total, siendo relacionado con más frecuencia con ureterocele o anomalías de inserción ureteral ectópica (Chacko et al.; Dorko et al.).

En mujeres, un uréter ectópico se abre para áreas fuera del control del músculo del esfínter, resultando en incontinencia urinaria e infecciones recurrentes. Si el examen de ultrasonido no revela malformaciones congénitas asociadas con uréter duplicado, los pacientes pueden desa- rrollar infección progresiva acompañada de dolor abdominal o sintomas de incontinencia ureteral (Dorko et al.).

Otra disfunción que puede ocurrir por duplicación total del uréter es el reflujo vesicoureteral, generalmente del uréter del polo inferior. Además de eso, el uréter del polo superior puede ser ectópico o tener un ureterocele asociado (Lashley et al., 2001). La mayoría de los casos requiere de correción quirúrgica (Kaplan et al., 1978; Ahmed \& Boucaut, 1988).

Otra complicación derivada de la duplicación total del uréter, ectópica o no, se puede dar en los transplantes renales. Alberts et al. han alertado que las complicaciones del transplante del uréter son la causa más importante de morbilidad quirúrgica después del transplante renal y la presencia de una duplicación ureteral en el injerto renal puede resultar en un aumento de la tasa de complicaciones. Debido a esto, existe cierto recelo por parte de algunos clínicos sobre el transplante de esos riñones. Sin embargo, debido a la escasez de donadores, los critérios para inclusión de éstos, que tienen contraindicaciones relativas, son cada vez más consideradas.

La contribución de la morfologia y la cooperación entre la urologia, diagnóstico por imágenes y especialistas en neonatología es escencial para determinar diagnóstico y tratamiento con base en el desarrollo de los síntomas clínicos de la enfermedad y la disfunción de los órganos (Dorko et al.).

El significado de este tipo de información morfológica busca complementar el conocimiento especializado de estas estructuras del sistema urinario, para su uso en la práctica diaria de clínicos especialistas en el área. Defectos en el desarrollo y anomalías asociadas a la duplicación del uréter y los riñones deben ser considerados en los diagnósticos diferenciales para evitar complicaciones y reducir la mortalidad.

GARBELOTTI JUNIOR, S.A.; RODRIGUES PEREIRA, V.; MARQUES, S. R.; MORAES DE CARVALHO, L. O. \& OLAVE, E. Non-ectopic duplicity of the ureter: clinical implications. Int. J. Morphol., 35(2):611-614, 2017.

SUMMARY: Duplication of the urinary tract is the most frequent variation of this system and may present as complete or incomplete duplication, as well as ectopic insertion throughout all parts of the urinary system from distal to the bladder. This article presents a complete non-ectopic duplication of the ureter in the 
left kidney of a cadaver of one Brazilian individual of male sex. In this study, both ureters originated in the renal hilum and continued to the urinary bladder separately, opening into different ostia, in the area of the bladder trigone, where the ostium belonging to the ureter that draining the upper pole presented a distal and lateral position in relation to the ostium of the ureter draining the lower pole. This arrangement is an exception to the Weigert-Meyer rule, which indicates that the ureter of the upper pole, due to its longer fixation to the mesonephric duct, presents a greater migration, ending medial and inferior to the ureter draining the inferior pole in $97 \%$ of cases.

KEY WORDS: Anatomy; Urinary system; Ureter duplication.

\section{REFERENCIAS BIBLIOGRÁFICAS}

Ahmed, S. \& Boucaut, H. A. Vesicoureteral reflux in complete ureteral duplication: surgical options. J. Urol., 140(5 Pt. 2):1092-4, 1988.

Alberts, V. P.; Minnee, R. C.; van Donselaar-van der Pant, K. A.; Bemelman, F. J.; Zondervan, P. J.; Laguna Pes, M. P. \& Idu, M. M. Duplicated ureters and renal transplantation: a case-control study and review of the literature. Transplant. Proc., 45(9):3239-44, 2013.

Chacko, J. K.; Koyle, M. A.; Mingin, G. C. \& Furness III, P. D. Ipsilateral ureteroureterostomy in the surgical management of the severely dilated ureter in ureteral duplication. J. Urol, 178(4 Suppl.):1689-92, 2007.

Chao, C. T. Ureteropelvic duplication as focus of recurrent infection. $Q . J$. M., 106(5):471-2, 2013.

Choi, H. \& Oh, S. J. The management of children with complete ureteric duplication: selective use of uretero-ureterostomy as a primary and salvage procedure. $B$. J. U. Int., 86(4):508-12, 2000.

Dorko, F.; Tokarcík, J. \& Viborná, E. Congenital malformations of the ureter: anatomical studies. Anat. Sci. Int., 91(3):290-4, 2016.

Ellerkamp, V.; Szavay, P.; Luithle, T.; Schäfer, J. F.; Amon, O. \& Fuchs, J. Single-stage surgical approach in complicated paediatric ureteral duplication: surgical and functional outcome. Pediatr. Surg. Int., 30(1):99-105, 2013.

Faion, A. G.; Castro Júnior, H. P.; Pena, G. F.; Silva, A. T. P. B. \& Abdo, R. P. B. Megaureter quadruplicado associado à duplicação ureteral contralateral: relato de caso. Rev. Méd. Minas Gerais, 13(4):290-1, 2003.

Goss, C. M. Gray Anatomia. Rio de Janeiro, Guanabara Koogan, 1988.

Kaplan, W. E.; Nasrallah, P. \& King, L. R. Reflux in complete duplication in children. J.Urol., 120(2):220-2, 1978.

Lam, H. S. Bifid blind-ending ureter a case report. Singapore Med. J., 32(1):84-6, 1991.

Lashley, D. B.; McAleer, I. M. \& Kaplan, G. W. Ipsilateral ureteroureterostomy for the treatment of vesicoureteral reflux or obstruction associated with complete ureteral duplication. J. Urol., 165(2):552-4, 2001

Moore, K. L.; Dalley, A. F. \& Agur, A. M. R. Anatomia Orientada para a Clínica. $7^{\mathrm{a}}$ ed. Rio de Janeiro, Guanabara-Koogan, 2014.

Senel, U.; Tanriverdi, H. I.; Ozmen, Z. \& Sozubir, S. Ectopic ureter accompanied by duplicated ureter: Three cases. J. Clin. Diagn. Res., 9(9):PD10-2, 2015.

Standring, S. Gray's Anatomy. The anatomical basis of clinical practice. $41^{\text {th }}$ ed. Edinburgh, Churchill Livingstone Elsevier, 2015.

Urdangarain, O. O.; Pérez, J. A. H.; Montes de Oca, J. O.; Rosales, F. M. \& García, C. R. Triplicación ureteral completa. Presentación de un caso. Arch. Esp. Urol., 59(3):284-7, 2006.

\author{
Dirección para correspondencia: \\ Dr. Enrique Olave \\ Facultad de Medicina \\ Universidad de La Frontera \\ Temuco \\ CHILE
}

E-mail: enrique.olave@ufrontera.cl

Recibido : 12-01-2017

Aceptado: 27-02-2017 